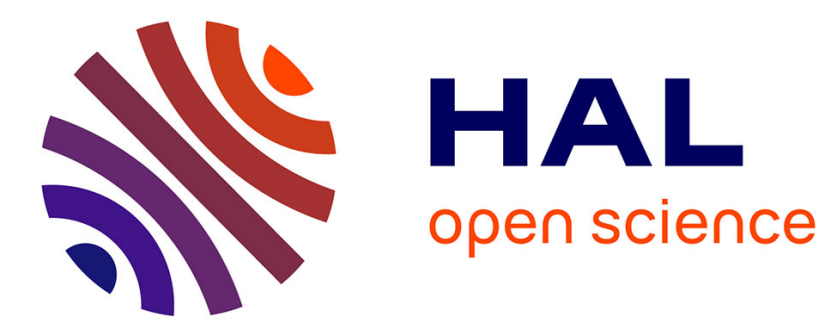

\title{
Model-based Adaptive Observers for Intake Leakage Detection in Diesel Engines
}

Riccardo Ceccarelli, Carlos Canudas de Wit, Philippe Moulin, A. Sciarretta

\section{To cite this version:}

Riccardo Ceccarelli, Carlos Canudas de Wit, Philippe Moulin, A. Sciarretta. Model-based Adaptive Observers for Intake Leakage Detection in Diesel Engines. ACC 2009 - American Control Conference, Jun 2009, Saint Luis, Missouri, United States. pp.1128-1133. hal-00438816v2

\section{HAL Id: hal-00438816 https://hal.science/hal-00438816v2}

Submitted on 13 Jun 2009

HAL is a multi-disciplinary open access archive for the deposit and dissemination of scientific research documents, whether they are published or not. The documents may come from teaching and research institutions in France or abroad, or from public or private research centers.
L'archive ouverte pluridisciplinaire HAL, est destinée au dépôt et à la diffusion de documents scientifiques de niveau recherche, publiés ou non, émanant des établissements d'enseignement et de recherche français ou étrangers, des laboratoires publics ou privés. 


\title{
Model-based Adaptive Observers for Intake Leakage Detection in Diesel Engines
}

\author{
Riccardo Ceccarelli , Carlos Canudas-de-Wit, Philippe Moulin and Antonio Sciarretta
}

\begin{abstract}
This paper studies the problem of diesel engine diagnosis by means of model-based adaptive observers. The problem is motivated by the needs of garantee highperformance engine behavior and in particular to respect the environmentally-based legislative regulations. The complexity of the intake systems of this type of engine makes this task particularly arduous and requires to constantly monitor and diagnose the engine operation. The development and application of two different nonlinear adaptive observers for intake leakage estimation is the goal of this work. The proposed model-based adaptive observers approach allows estimating a variable that is directly related to the presence of leakage, e.g., hole radius. Monitoring and diagnostic tasks, with this kind of approach, are straightforward. Two different approaches, whose main difference is on observer adaptation law structure are studied. One approach is based on fixed gains while the other method has variable gain. The paper also includes a comparative study of the two methods in simulations using advanced diesel engine professional simulator AMEsim.
\end{abstract}

\section{INTRODUCTION}

Modern diesel engine has the potential of a significant reduction of pollutant emissions with respect to more conventional engines. In particular, the air system is a complex system that typically includes throttle actuators, variable geometry turbochargers, exhaust gas recirculation, and several sensors. The proper system operation is strongly dependent on the correct functioning of all these subsystems. In order to keep high performance of the air system, monitoring tasks such as leakages at differents locations, the detection of malfunctioning of sensors and actuators (i.e. EGR valve opening), as well as those imposed by environmentally-based legislative regulations are necessary.

A promising way to enhance monitoring and diagnosis systems is to adopt model-based techniques, which have been used in different engine frameworks [14], [10], [12] [6], [8]. See also [10] for a survey. Physical models of intake receiver can be found in [15] where a deep description and analysis of the functioning of a air-path in a diesel engine with exhaust gas recirculation circuit is presented. More physical air-path modeling, including conservation laws applied to the receiver, flows modeling can be found in [4],[5].

An interesting approach to model-based air-path faults detection can be found in [6] and in [8]. By using several

R. Ceccarelli (corresponding author) is a PhD Candidate in Automatic Control at GIPSA-lab, Institut National Polytechnique de Grenoble, 961, rue de la Houille Blanche Domaine universitaire - BP 46 F - 38402 Saint Martin d'Hères, France riccardo. ceccarelli@ifp.fr

C. Canudas-de-Wit is with the CNRS at the Control System Department of GIPSA-lab carlos. canudas-de-witeinpg.fr

P. Moulin and A. Sciarretta are with the Department of Engine Control in IFP, 1 et 4 Avenue de Bois Preau, 92852 Rueil-Malmaison, France models in parallel, each one sensitive to one kind of fault, predicted outputs is compared and a diagnosis is provide. In particular, hypothesis test methodology proposed in [6] deals with the multi-fault detection in air-path system. In [8] the authors propose an extended adaptive Kalman filter to find which faulty model best matches with measured data, then a structured hypothesis allows going back to the faults.

Model-based adaptive observers represent another alternative strategy for intake leakage detection and estimation. Adaptive observers allow not only to estimate signals that are not available from direct measurement, but also some of the unknown model parameters. In some cases, as here in problem, the unknown model parameters are directly associated to the fault to be detected, and the observer can also track small variations of such parameters.

In this paper two class of adaptive observers are studied. Adaptive observers with fixed gains similar to the ones used to estimate tire/road contact friction in [1], [2], [3], are here studied and redesigned to problem at hand. These nonlinear observers are designed based on Lyapunov functions. Their convergence is asymptotic and their structure and tuning is relatively simple. A more complex strategy applied here is based on variable-gain observers along the lines proposed by [13]. This leads to observers that may have (under certain assumptions) exponential convergence, although they are more computational involved.

In this paper the performance of the two classes of adaptive nonlinear observers by using them in a fault detection and identification (FDI) framework are compared. Their application to the detection of intake leakage in a Diesel engine air path, when no more sensors than those available in standard mass-production vehicles engines are added, is studied. The use of these type of observers represent a good strategy for this specific FDI problem.

The paper is organized as follows. A reference model is presented in Section II. Section III presents two different types of observers, with fixed gain and variable gain respectively. Persistent condition for parameter estimation is discussed with respect to leakage hole diameter estimation. Section IV points out the differences in the application of these two nonlinear adaptive observers for intake leakage detection and estimation. In particular, threshold determination for fault detection is investigated and some simulation results for $4 \mathrm{~mm}$ hole diameter are shown.

\section{SYSTEM DESCRIPTION}

The Diesel engine considered in this paper is a fourcylinders engine with a high-pressure exhaust gas recircu- 
lation circuit (EGR) as described in [15]. The purpose of this section is to propose a simpler rapresentation of the engine in order to design an observer. A description of engine and justification of some simplification is done in [17]. A schematic picture of the air intake system is shown in Figure (1).

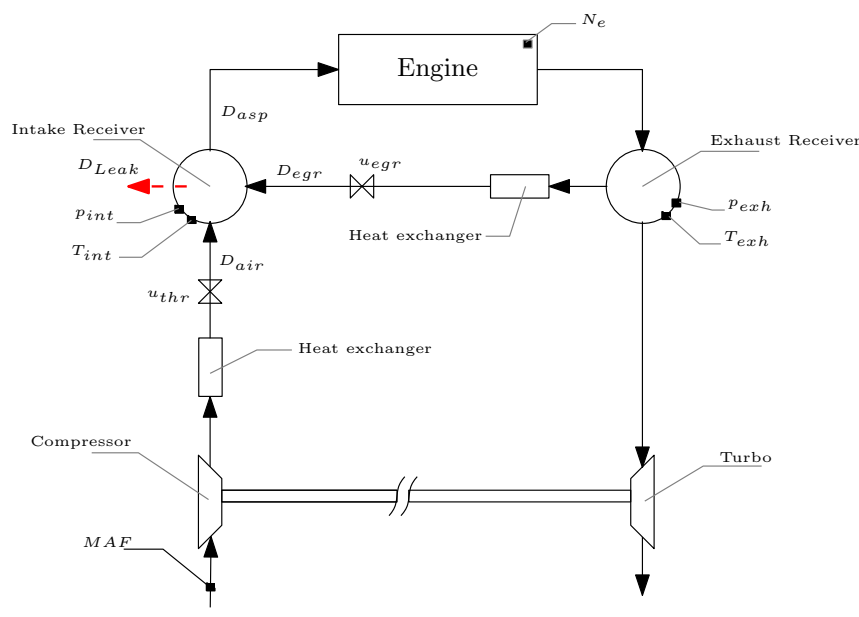

Fig. 1. A schematic picture of the air-intake system.

Ambient air enters in the intake receiver through the compressor and its flow rate $D_{\text {air }}$ is measured by a mass air flow sensor. Fresh air is mixed in the intake receiver with exhaust gas coming from the exhaust receiver $\left(D_{\text {egr }}\right)$ and then aspirated $\left(D_{a s p}\right)$ by the engine that is seen as a volumetric pump. The intake volume is assumed as small enough to approximate the process as isothermal. Under previous assumption, the intake pressure $p_{\text {int }}$ is the only state variable, whose dynamics is

$$
\dot{p}_{i n t}=\alpha_{i n t}\left(D_{a i r}+D_{e g r}-D_{a s p}-D_{\text {Leak }}\right),
$$

where $\alpha_{i n t}=R_{\text {air }} T_{\text {int }} / V_{\text {int }}, R_{\text {air }}$ is the universal gas constant, $V_{\text {int }}$ is the intake volume, $T_{i n t}$ is the measured intake temperature, $D_{\text {Leak }}$ is the leakage mass flow rate from the intake in presence of a hole. All flows are modeled by the following generic equation of flow through an orifice,

$$
D=S \frac{p_{\text {in }}}{\sqrt{R T_{\text {in }}}} \sigma\left(\frac{p_{\text {out }}}{p_{\text {in }}}\right)
$$

where

$$
\sigma\left(\frac{p_{\text {out }}}{p_{\text {in }}}\right)= \begin{cases}\frac{1}{\sqrt{2}} & \text { if } \quad \frac{1}{2} p_{\text {in }} \geq p_{\text {out }} \\ \sqrt{2 \frac{p_{\text {out }}}{p_{\text {in }}}\left(1-\frac{p_{\text {out }}}{p_{\text {in }}}\right)} & \text { if } \quad \frac{1}{2} p_{\text {in }} \leq p_{\text {out }}\end{cases}
$$

and where the subscripts " $i n$ " and "out" stand for upstream and downstream values across a (possibly variable-area) section $S$.

Equation (1) can be re-written as a first general structure:

$$
\begin{aligned}
\dot{x} & =-a(x, z) x-\psi(x, z) \theta+\phi(x, z) \\
\dot{\theta} & =0 \\
y & =x
\end{aligned}
$$

where the scalar state $x(t)=p_{\text {int }}$ and $z \in \Re^{7}$ is a vector containing variables that can be measures (see TABLE I for details). Finally, $\theta$ is the parameter to be estimated,that is, the percentage of the maximal leakage (7) such that $D_{\text {Leak }}=$ $\theta \cdot \psi(x, z)$. By the following $y(t)$ and $x(t)$ will be used without distiction. Moreover

$$
\begin{aligned}
a(x, z) & =\alpha_{i n t} \beta_{i n t} \eta_{v}\left(x, N_{e}\right) \\
\psi(x, z) & =\alpha_{\text {int }} S_{\max } \frac{x}{\sqrt{R T_{i n}}} \sigma\left(\frac{p_{a m b}}{x}\right) \\
\phi(x, z) & =\alpha_{\text {int }}\left(D_{\text {air }}+D_{\text {egr }}\right)
\end{aligned}
$$

where $\psi(x, z)$ is the gas mass flow rate through the hole when $S=S_{\max }, a(x, z) x$ is the flow $D_{a s p}$ aspirated by the engine is considered as a volumetric pump, $\eta_{v}\left(x, N_{e}\right)$ is the engine volumetric efficiency, generally tabulated as a function of intake pressure and engine speed, and

$$
\beta_{\text {int }}=\frac{1}{R T_{\text {int }}} V_{c y l} \frac{N_{e}}{120} .
$$

where $V_{c y l}$ is the total displacement volume.

The EGR flow $D_{e g r}$ is computed by using the avalaible measures of $p_{\text {exh }}, T_{\text {exh }}, p_{\text {int }}, u_{\text {egr }}$ in equation (2). By injecting the measured state $y(t)$ in $a, \psi$ and $\phi$, equation (3) can be re-written as

$$
\dot{x}=-a(y, z) x-\psi(y, z) \theta+\phi(y, z)
$$

where $a, \psi$ and $\phi$ are now completely known functions. Intake receiver model (9) can be finally presented in a more general and compact form as

$$
\left\{\begin{array}{l}
\dot{x}=-a(t) x-\psi(t) \theta+\phi(t) \\
\dot{\theta}=0 \\
y=x
\end{array}\right.
$$

Note that the original system model (1) has been extended with the additional equation (4), which implicitly assumes that this parameter is constant. It is also straightforward to note that $\theta$ is observable from $y$ as long as $\psi(t) \neq 0$. Hence observability of $\theta$ is preserved even if $\psi(t)=0$ only at a finite numbers of points in time.

TABLE I

EXTERNAL VARIABLES

\begin{tabular}{|c|c|c|c|}
\hline Variable name & Meas. name & Description & Units \\
\hline \hline$z_{1}$ & $D_{\text {air }}$ & Mass air flow & {$[\mathrm{kg} / \mathrm{s}]$} \\
$z_{2}$ & $T_{i n t}$ & Intake temperature & {$[\mathrm{K}]$} \\
$z_{3}$ & $p_{\text {exh }}$ & Exhaust pressure & {$[\mathrm{Pa}]$} \\
$z_{4}$ & $T_{\text {exh }}$ & Exhaust temperature & {$[\mathrm{K}]$} \\
$z_{5}$ & $u_{e g r}$ & Egr valve position & {$[\%]$} \\
$z_{6}$ & $u_{t h r}$ & Throttle valve position & {$[\%]$} \\
$z_{7}$ & $N_{e}$ & Engine speed & {$[\mathrm{rad} / \mathrm{s}]$} \\
\hline
\end{tabular}

The rest of the paper is now devoted to design two classes of observers to estimate $\theta$ based on the extended model (10).

\section{OBSERVER DESIGN}

Fault detection techniques are based on the design of some residual terms. They are signals, normally equal to zero, which change their values only in the presence of faults. The 
natural residual for leakage detection is the error between the intake pressure measured, $y$, and estimated from (3), $\hat{y}$. Another variable of interest is the estimated parameter $\hat{\theta}$ itself. If there is no fault, the diameter of hole should be equal to zero (or small if noise is present). It is sensibly different from zero only if a leakage is present. Here two different nonlinear adaptive observers for joint intake pressure and leakage estimation are presented.

\section{A. Fixed-gain observer}

The first proposed observer is a Lyapunov-based designed. The proposed structure is:

$$
\left\{\begin{aligned}
\dot{\hat{x}} & =-a(t) \hat{x}-\psi(t) \hat{\theta}+\phi(t)+K_{0}(y-\hat{x}) \\
\dot{\hat{\theta}} & =\xi(t, \hat{x})
\end{aligned}\right.
$$

where $K_{0}>0$ is the gain influencing the pressure estimation convergence rate, and $\xi(t, \hat{x})$ is the adaptation law to be designed. The error system equations in terms of the error variables: $\tilde{x}=x-\hat{x}=y-\hat{x}$ and $\tilde{\theta}=\theta-\hat{\theta}$ is:

$$
\left\{\begin{array}{l}
\dot{\tilde{x}}=-a(t) \tilde{x}-\psi \tilde{\theta}-K_{0} \tilde{x} \\
\dot{\tilde{\theta}}=-\dot{\hat{\theta}}=-\xi(t, \hat{x})
\end{array}\right.
$$

Consider the following scalar Lyapounov function

$$
V=V(\tilde{x}, \tilde{\theta})=\frac{1}{2} \tilde{x}^{2}+\frac{1}{2 \gamma} \tilde{\theta}^{2}
$$

Its time-derivative along the error equation dynamics is given by

$$
\dot{V}=-a(t) \tilde{x}^{2}-K_{0} \tilde{x}^{2}-\psi \tilde{\theta} \tilde{x}+\frac{1}{\gamma} \tilde{\theta} \xi(t, \hat{x})
$$

If the adaptation law $\xi(t, \hat{x})$ is designed to cancel the last two term in $\dot{V}$, i.e.,

$$
\xi(t, \hat{x})=-\gamma \psi(t) \tilde{x}=-\gamma \psi(t)(y-\hat{x})
$$

and moreover $a(t)>0 \forall t$ and a $K_{0}>0$ is choosen, then

$$
\dot{V}=-\left(K_{0}+a(t)\right) \tilde{x}^{2} \leq 0 .
$$

From standard Lyapunov and invariance set arguments, $\tilde{x}$ will converge to zero asymptotically. Also, from the error equation (12) the following limit should hold

$$
\lim _{t \rightarrow \infty} \psi(t) \tilde{\theta}=0
$$

Therefore, if $\lim _{t \rightarrow \infty} \psi(t) \neq 0$, also $\lim _{t \rightarrow \infty} \tilde{\theta}=0$. Note that condition $\lim _{t \rightarrow \infty} \psi(t) \neq 0$ is the observability condition, which is always fulfilled, unless the intake pressure approaches the ambient pressure. This situation may occur in two different ways:

- A large diameter hole appears in the receiver, leading to a decrease of the intake pressure until it reaches $p_{a m b}$.

- For some engine operation points intake pressure is controlled to be close to $p_{a m b}$. In this case observability condition for parameter $\hat{\theta}$ is weak.

Summarizing, the following theorem applies
Theorem 3.1: Consider the nonlinear adaptive observer:

$$
\left\{\begin{aligned}
\dot{\hat{x}} & =-a(t) \hat{x}-\psi(t) \hat{\theta}+\phi(t)+K_{0}(y-\hat{x}) \\
\dot{\hat{\theta}} & =-\gamma \psi(t)(y-\hat{x})
\end{aligned}\right.
$$

with positive constants $\gamma$ and $K_{0}$. This observer, applied to the system under consideration, has the following properties:

$i$ ) The error signals $\tilde{x}$ and $\tilde{\theta}$ are bounded

ii) $\lim _{t \rightarrow \infty} \tilde{x}=0$

iii) In addition, if $\lim _{t \rightarrow \infty} \psi(t) \neq 0$, then

$$
\lim _{t \rightarrow \infty} \tilde{\theta}=0
$$

The stability and convergence rate are tuned by the $\gamma$ and $K_{0}$.

\section{B. Variable-gain observer}

An interesting adaptive observer for state-affine nonlinear systems was proposed in [13] and here it is adapted for leakage detection and estimation.

1) Zhang observer design: The general system class considered by Zhang [13] comprises state-affine nonlinear systems of the form

$$
\left\{\begin{aligned}
\dot{x} & =a(t) x+b(t) u+\psi(t) \theta+\phi(t) \\
\dot{\theta} & =0 \\
y & =c(t) x
\end{aligned}\right.
$$

which are particularized here to scalar signals. For such kind of systems it is possible to design an exponentially convergent adaptive observer to jointly estimate $x$ and $\theta$ under the persistent excitation conditions given next.

Assumption 3.1: Assume that the pair $(a(t), c(t))$ in system (15) is such that a bounded time-varying gain $K(t)$ exists so that the system

$$
\dot{\eta}=[a(t)-K(t) c(t)] \eta
$$

is globally exponentially stable.

Assumption 3.2: Let $\Gamma(t)$ be a function generated by the ODE system

$$
\dot{\Gamma}=[a(t)-K(t) c(t)] \Gamma+\psi(t)
$$

Assume the $\psi(t)$ is persistently exciting (PE) so that two positive constants $\delta, T$ exists such that $\forall t>0$ the following inequality holds

$$
\int_{t}^{t+T}|\Gamma(\tau) c(\tau)| d \tau \geq \delta I
$$

Under these assumptions the following theorem is stated

Theorem 3.2: [13] Let $\Lambda>0$ be any positive gain. Under Assumption 3.1 and 3.2 and for constant $\theta$, the ODE system

$$
\left\{\begin{aligned}
\dot{\hat{x}}= & a(t) \hat{x}+b(t) u+\psi(t) \hat{\theta}+\phi(t)+\ldots \\
& \ldots+\left[K(t)+\Lambda \Gamma^{2} c(t)\right][y-c(t) \hat{x}] \\
\dot{\hat{\theta}}= & \Lambda \Gamma c(t)[y-c(t) \hat{x}] \\
\dot{\Gamma}= & {[a(t)-K(t) c(t)] \Gamma+\psi(t) }
\end{aligned}\right.
$$

is a global exponential adaptive observer for system (15), i.e. for any initial conditions the observation errors $\hat{x}(t)-x(t)$ and $\hat{\theta}(t)-\theta$ tend to zero exponentially fast when $t \rightarrow \infty$ 
2) Application to the intake receiver model: The structure of the system considered in section II (eqn. 10 closely resembles that of (eqn. 15) thus is possible to apply the Zhang observer for jointly estimation of intake pressure and leakage hole diameter. With this aim, the observer used for fault detection and estimation proposed it has the following structure,

$$
\left\{\begin{aligned}
\dot{\hat{x}} & =-a(t) \hat{x}+\phi(t)-\psi(t) \hat{\theta}+\left(K_{0}+\Lambda \Gamma^{2}\right)[y-\hat{x}] \\
\dot{\hat{\theta}} & =\Lambda \Gamma[y-\hat{x}] \\
\dot{\Gamma} & =\left[-a(t)-K_{0}\right] \Gamma-\psi(t)
\end{aligned}\right.
$$

where $\Lambda$ is a tuning gain.

In the following assumptions (3.1) and (3.2) are correctly verified.

Assumption 3.1 is always verified because of the nature of the system: volumetric efficency is always a positive quantity and thus the aspiration process is inherently stable. Therefore, it suffices that $K(t)>-a(t) \forall t$ to satisfy Assumption 3.1. For instance, a constant $K(t)=K_{0}>0$ equal to the value choosen for the fixed-gain observer (11), i.e., in order make a easier comparation between these two observers. As proposed in [13] a optimal Kalman filter gain can be used as well.

Assumption 3.2, as already discussed in section III-A, is on persistent eccitation condition of leakage function $\psi(t)$ which depends on the valve pressure ratio across the valve. As $\psi(t)$ represents the maximal leakage flow for each operation condition, then $\psi(t)$ is equal to zero only if the intake pressure reaches the ambient pressure. As shown in section IV-B the estimation of $\theta$ is physically difficult or even impossible for some specific operative point, where intake pressure is close to ambient pressure. This is a generic condition for this physical system (observability condition), that is independent of the observer design.

As it has been detailed in the observer design of section III-A, if the PE condition is not satisfied, $\tilde{\theta}$ can not be ensured to tend to zero, although the prediction error $\tilde{x}$ does.

\section{MOdEL IN THE LOOP: RESUltS}

This section is devoted to the intake leakage detection and the hole diameter estimation by the use of the two studied observers. Experimental setup and hypothesis used for simulation are introduced in IV-A. In IV-B threshold determination for leakage detection is discussed. A comparative analysis of the two observers in presence of a hole on the receiver, supported by simulation results, is the subject of section IV-C.

\section{A. Experiment description and diagnosis setup}

Fault detection and identification (FDI) algorithms, based on the adaptive observers (11) and (20) have been tested on a four cylinders Diesel engine model running on AMEsim $\mathbb{R}$ platform in cosimulation with Mathworks Matlab $\cap$. The AMEsim model used for simulation has been validated on engine testbed present at IFP [15]. In the following of this paper the AMEsim model is called reference model. Note that its dynamic is more complex than the model (eqn. 10) described for observer design. Differences between model and reference model lead to some errors in simulation. Here some possible causes of error:

- some supposed perfectly known variables are obtained by function of vector $z(t)$, e.g., $D_{\text {egr }}$ is not directly available but computed by equation (2) using $y(t)$ and $z_{3}$. Equation (2) accurancy, as it will be discussed in section IV-B, depends on pressure ratio (i.e. $p_{\text {int }} / p_{\text {exh }}$ ) across the valve;

- In model (10) heat exchangers volume and pipes volumes are neglected;

- $D_{\text {air }}$ is obtained by $M A F$ sensor which is positioned (see fig. 1) at inlet of compressor. $M A F$ sensor give a good measure of $D_{\text {air }}$ when system is stabilized but, during the transient, the measure differs from the flow entering in the intake manifold due to dynamic into volume;

- the volumetric efficency $\eta_{v}$ in function $a(t)$ is obtained from a lookup table which differs from real instantaneous value and depends on engine operating point. This can lead to error on real estimation of the aspirated gas into cylinders.

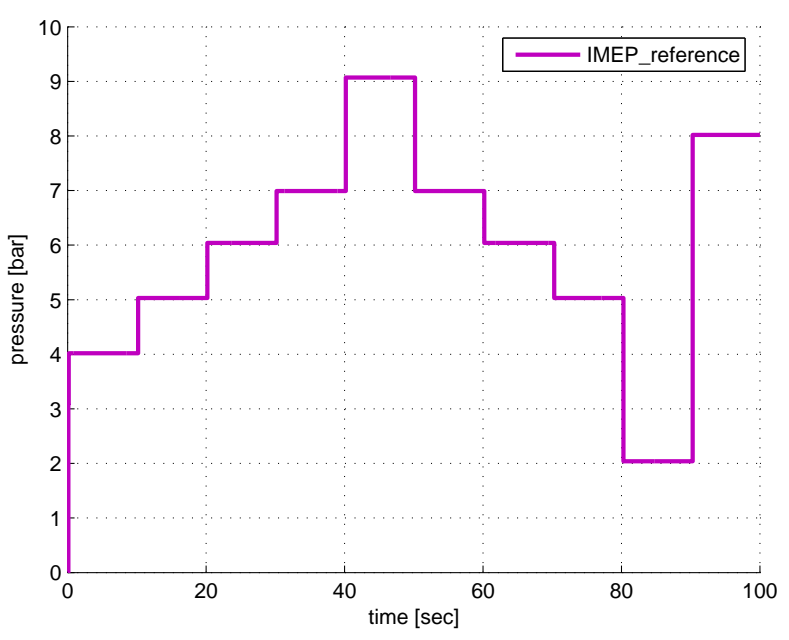

Fig. 2. IMEP - load transient trajectory.

All co-simulation tests shown have been done on load transients at $1500 \mathrm{rpm}$, i.e., each load is kept for 10 seconds (fig. 2). Modelling errors, described before, will act on parameter estimation of the hole diameter by a time-varing bias depending on engine IMEP working zone (fig. 2).

The physical values used in model are reported in Table (II).

\section{B. Simulation without leakage: threshold determination}

In section III some remarks on PE condition for $\hat{\theta}$ convergence have been done, particular attention was paid to the function $\psi(t)$ which must differ from zero to garantee hole diameter estimation. 
TABLE II

MODEL VALUES FOR SIMULATIONS

\begin{tabular}{|c|c|c|c|}
\hline Name & Value & Units & Description \\
\hline \hline$V_{\text {int }}$ & $0.8 e-3$ & $m^{3}$ & Intake Volume \\
$R$ & 287 & $\mathrm{~J} / \mathrm{K}$ & Universal gas costant \\
$S_{\max }$ & $1.5904 e-3$ & $\mathrm{~m}^{2}$ & Maximal Leakage Area \\
$S_{\max , \text { egr }}$ & $411.607 e-6$ & $\mathrm{~m}^{2}$ & Maximal EGR Section \\
$S_{\max , t h r}$ & $1250 e-6$ & $\mathrm{~m}^{2}$ & Maximal Throttle Section \\
\hline
\end{tabular}

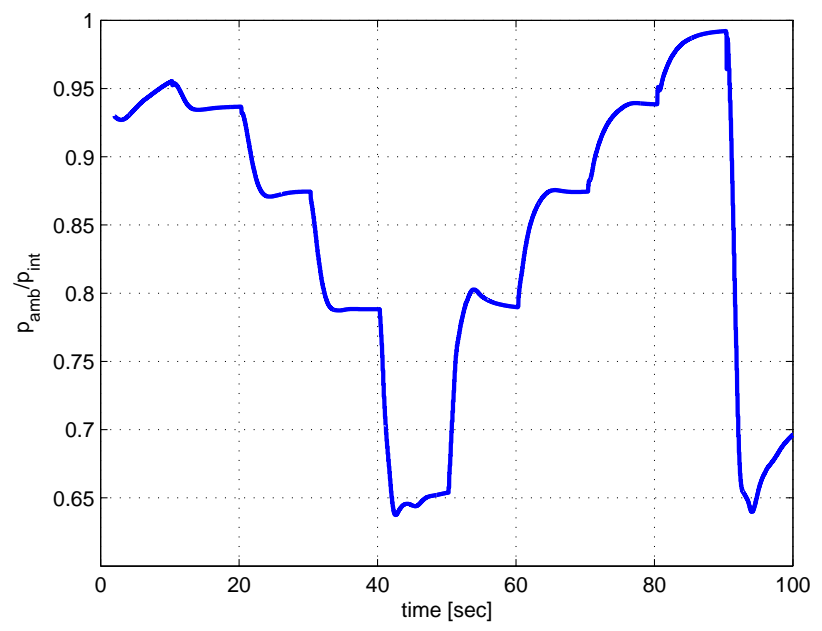

Fig. 3. Pressure ratio $p_{a m b} / p_{\text {int }}$ during a transient load at $1500 \mathrm{rpm}$.

The function $\psi(t)$ (eqn. 7) can go to zero only if $\sigma\left(p_{a m b} / p_{\text {int }}\right)$ go to zero as well, i.e. $p_{r}=1$. For this reason the pressure ratio $p_{r}=p_{a m b} / p_{\text {int }}$ is studied. In figure (3) is shown the evolution of $p_{r}$ during a load transient (fig. 2) when no hole is present on intake receiver. For some operating points the pressure ratio is close to one, which imply $\psi(t) \approx 0$. For these operation points $\hat{\theta}$ estimation becomes more difficult (i.e. estimation between $[0,10]$ and $[80,90]$ seconds). In presence of an hole, $p_{r}$ would be even higher than that shown in figure (3) all along the IMEP trajectory, which imply that the PE condition is more difficult to be checked.

For threshold level determination, the estimation of $\hat{\theta}$ (i.e. percentage of $D_{\text {Leak, max }}$ ) made by the two studied observers is monitored when there is no hole.

In figure (4) a comparison of the $\hat{\theta}$, obtained by the two observers, is shown. As there is no leakage, the expected estimation should be approximately zero all along the simulation. Particular attention have to be paid to intervals $[0,10]$ sec. and $[80,90]$ sec where $p_{r}>0.9$. In such intervals a weak PE condition is verified and so the parameter estimation convergence is not garantee (fig. 4) before the change of IMEP reference.

Moreover, all previously discussed modelization errors, affect the estimation of the hole diameter and depends on operating point. For instance, in interval $[50,70]$ sec. the estimation is matching the real diameter of the hole (i.e. $0 \mathrm{~mm}$ ).

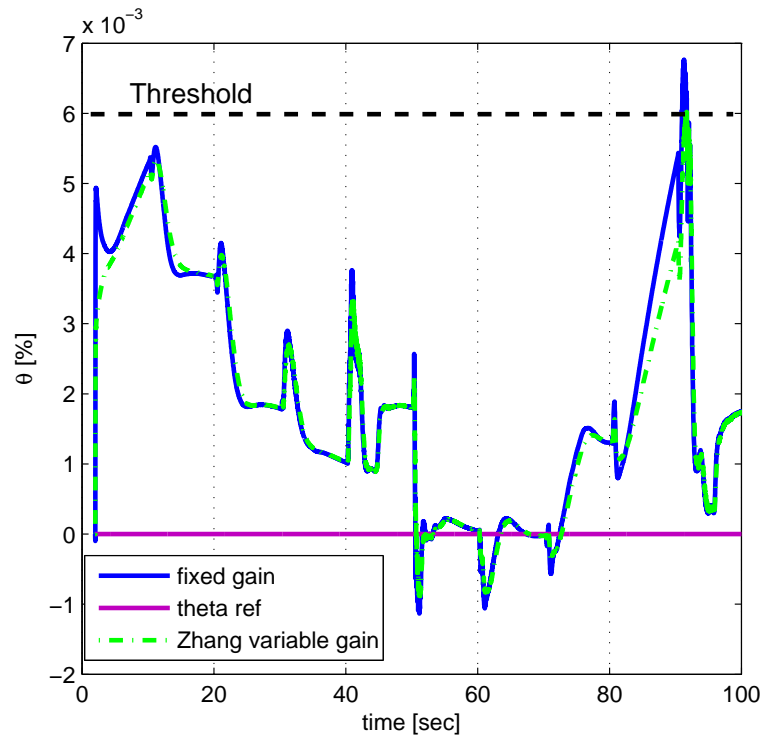

Fig. 4. No leakage - $\hat{\theta}$ estimated by the two observers during a transient load at $1500 \mathrm{rpm}$.

A possible choice threshold level determination for leakage detection is to fix at 0.006 to avoid possible false alarm. Other choices can be done, for instance a variable threshold rely to the operating point can be chosen.

By choosing threshold equal to 0.006 only hole diameter greater than $4 \mathrm{~mm}$ can be detected and estimated.

C. Comparative behaviour of the two observers

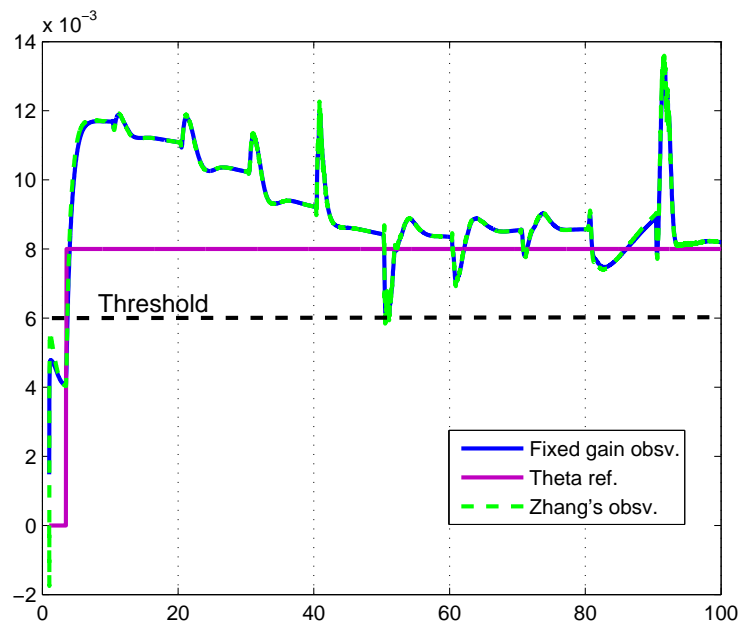

Fig. 5. 4mm fault estimation comparison.

Figure (5) shows the detection and estimation obtained by the two observers (11) and (20) when a hole of $4 \mathrm{~mm}$ (i.e. $0.008 \%$ of $S_{\max }$ ) of diameter is present on intake receiver. The estimation maximal error made is of $1 \mathrm{~mm}$ more the correct value (i.e. $5 \mathrm{~mm}$ estimated hole diameter). Moreover 
error decreases as pressure ratio $p_{r}$ is far from one (see fig. 3): in interval from 50 to 70 seconds the hole diameter estimation is relatively close to the real hole diameter. Spikes can be reduced by decreasing the state observer gain $K_{0}$.

Observers parameter estimation seems to have a very close dynamic all along the transient load trajectory, a more precise analysis is subject of the following paragraphs.

1) Adaptive gain analysis: Fixed-gain and Zhang's observers, as it possible to be seen in fig. 4 and 5, have a very close behaviour. The variable gain of Zhang's observer $\Gamma(t)$ (see eqn. 20) acts on the speed convergence of the parameter and state estimation.

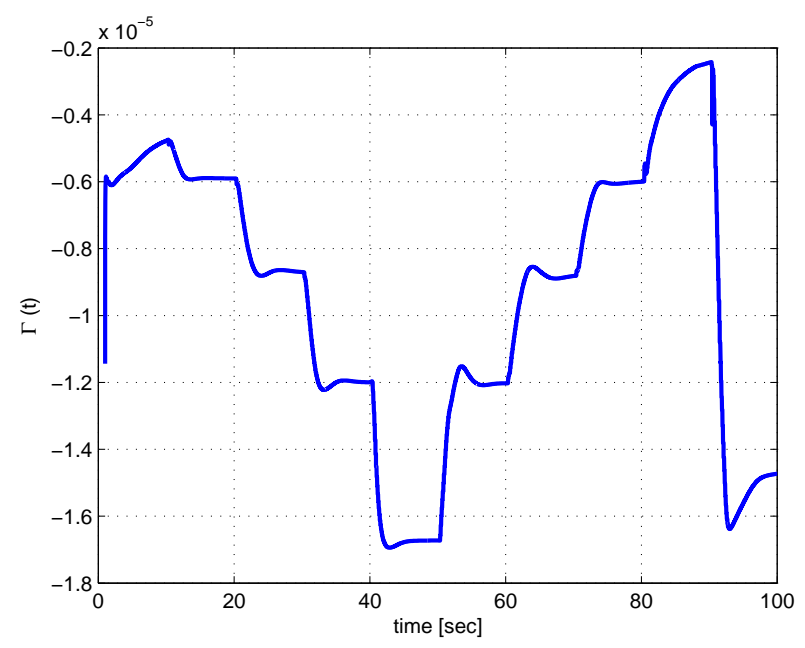

Fig. 6. $\quad \Gamma(t)$ during a transient load at $1500 \mathrm{rpm}$ when a leakage hole of $4 \mathrm{~mm}$ is present

By analysing the variable gain $\Gamma(t)$ (fig. 6) in steady-state condition, it becomes

$$
\Gamma_{s s}=\frac{\psi_{s s}}{a_{s s}+K_{0}}
$$

where subscript $s s$ stands for steady-state. By comparison of the two adaptive laws, the $\Gamma$ term in Zhang observer has the same role of the $\psi$ in (14), so the difference between them, as $K_{0}$ is supposed constant and the same for the two observers, rely on the variation of $a_{s s}$. In the studied case, the term related to volumetric efficiency, i.e. $a(t)$, is in general negligible with respect of $K_{0}$ and $\psi(t)$.

2) State convergence in Zhang's Observer: In the previous section the effect of $\Gamma(t)$ on parameter estimation has been analysed. The variable gain $\Gamma(t)$ acts also on state estimation, as expressed in the first equation of system (20). As seen before (fig. 6) $\Gamma(t)$ is small, this means that the correction term $\Lambda \Gamma^{2}$ does not act any important contribution in the state estimation convergence (i.e. $\Gamma^{2} \approx 10^{-12}$ ).

\section{CONCLUSION}

In this paper two different observers for online detection and estimation of leakage in intake receiver have been presented. Particular attention has been payed to PE condition necessary for convergence of parameter estimation. For the problem proposed, even by choosing a fixed threshold, very small hole can be detected. The simulation comparation results lead to affirm that, for the considered problem, no significant differences can be pointed out in the behaviour of the two observers; the reasons of this matching have been investigated.

The fixed gain observer designed by Lyapounov constructive method has a simpler structure than the Zhang's observer. The simpler structure and a so close behaviour suggest the fixed-gain observer to be used in real application. However Zhang observer by its variable gain offers more customize tuning parameters and gives a general framework (structure and conditions) for the design of an adaptive observer for MIMO time-varying state-affine nonlinear systems which might be useful in future design of leakages detection and estimation in different parts of air-path system. Moreover, it garantees exponential convergence also in the case where parameter is not supposed constant but with a bounded derivative rate. This last point is very interesting in leakage detection which generally may be modeled, when hole occurs, as a discontinuity and after by an linear increasing (bounded rate) diameter.

\section{REFERENCES}

[1] Canudas-de-Wit, C., and R. Horowitz, (1999), Observers for Tire/road Contact Friction using only wheel angular velocity information., In 38th IEE-CDC 1999.

[2] Canudas de Wit, R. Horowitz, and P. Tsiotras, (1999), Model-based observers for tire/road contact friction prediction, In "New Directions in Nonlinear Observer Design", Nijmeijer, H. and T.I Fossen (Eds), Springer Verlag, Lectures Notes in control and Information Science, May 1999.

[3] Canudas-de-Wit C., M. L. Petersen M.L., and Shiriaev A. "A New Nonlinear Observer For Tire/Road Distributed Contact Friction". IEEE Control and Decision Conference, Mahui, USA, Dec. 2003.

[4] Guzzella, L., Onder, C.H., "Introduction to Modeling and Control of Internal Combustion Engine System", Springler-Verlag Berlin Heidelberg 2004

[5] Heywood, J., "'Internal Combustion Engine Fundamentals", McGrawHill, Inc, 1998

[6] Nyberg, M. "Model-Based Diagnosis of an Automotive Engine Using Several Types of Fault Models" IEEE Transactions on control systems technology, vol. 10, n 5, September 2002

[7] Nyberg, M., Perkovic, A., Nielsen, L. "Model Based Diagnosis of Leaks in the Air Intake System of An SI Engine" SAE paper 980514, 1998

[8] Nyberg, M., Sutte, T. "Model based diagnosis of the air path of an automotive diesel engine", Control Engineering Practice 12, pages 513-525, 2004

[9] Isermann, R. "Supervision, fault-detection and fault diagnosis methods - an introduction", Control Engineering Practice, vol. 5, pages 639652, 1997

[10] Isermann, R. "Model Based Fault Detection and Diagnosis Methods" Proc. of the American Control Conference, 1996

[11] Blanke, M. et al "Fault Detection for a diesel engine actuator - A benchmark for FDI" Control Engineering Practice, vol. 3, $\mathrm{n}^{\circ} 12$, pages 1731-1740, 1995

[12] Isermann, R., Schwarte, Kimmich "Model based fault detection of a diesel engine with turbocharger. A case study" Proceedings of the IFAC conference, 2004

[13] Zhang, Q., "Adaptive Observer for MIMO Linear Time Varying System", Rapport de recherche n.4111, INRIA, 2001

[14] Lee, B., Guezennec, Y., Rizzoni, G. "Model based fault diagnosis of spark ignitiondirect injection engine using nonlinear estimations", Proceedings of the SAE conference, 2005-01-0071, 2005

[15] Albrecht, A., J. Chauvin, J., Lafossas, FA, Potteau, S., and Corde, G. "Development of highly premixed combustion Diesel engine model : from simulation to control design" SAE paper 2006-01-1072 
[16] J., Chauvin, G., Corde, N., Petit and P., Rouchon "Experimental Motion Planning in Airpath Control for HCCI engine" in Proc. of the IEEE Conf. American Control Conference, 2006.

[17] J. Chauvin, J., Albrecht, A., Corde, G. and Petit, N., "Modelling and Control of a Diesel HCCI Engine" Fifth IFAC Symposium on Advanced in Automotive Control, ACC 2007 